\title{
Extraction of circuital parameters of organic solar cells using the exact solution based on Lambert W- function
}

\author{
G. Del Pozo, B. Romero, and B. Arredondo \\ Dpto. Tecnología Electrónica, Escuela Superior de Ciencias Experimentales y Tecnología, Universidad Rey Juan Carlos, \\ C\ Tulipán s\n, 28933, Móstoles, Madrid, Spain
}

\begin{abstract}
The electrical behavior of organic solar cell (OSC) has been analyzed using a simple circuital model consisting on an ideal diode together with a series and parallel resistances $\left(R_{S}\right.$ and $R_{P}$ respectively). Applying Kirchhoff's Laws to the circuit leads to a transcendental equation that can be solved numerically without approximations using the Lambert $\mathrm{W}$ function. Theoretical expression has been fitted to experimental current-voltage (I-V) curves under forward bias, obtaining fairly accurate values for the electrical parameters. This model has been validated comparing the extracted parameters for dark and illumination conditions of different devices. Results show good agreement for $R_{S}$, and ideality factor $(\eta)$.

Electrical parameters obtained in this work are also compared to those ones extracted using an approximated method often employed by other authors ${ }^{1}$. We conclude that approximated method leads to reasonable good values for $R_{S}, R_{P}$ and $\eta$. However, in the case of $R_{p}$ the voltage range chosen to fit the data with the exact method must be constrained to the fourth quadrant, where the role of parallel resistance is more critical.

To validate the model, a bunch of organic solar cells with structure ITO/ poly(3,4-ethylenedioxythiophene)-poly (4styrene sulfonate (PEDOT:PSS)/ poly(3-hexylthiophene) (P3HT): 1-(3-methoxycarbonyl)-propyl-1-1-phenyl-(6,6)C61 (PCBM)/Al has been fabricated in inert atmosphere. Different active layers were deposited varying the P3HT:PCBM ratio $(1: 0.64,1: 1,1: 1.55)$ and the active layer thickness (ranging from 100 to $280 \mathrm{~nm}$ ). Devices are encapsulated inside the glove-box prior its characterization outside the glove-box. Electro optical characterization has been performed with a halogen lamp.
\end{abstract}

Values extracted for $R_{S}$ range from $142 \Omega$ to $273 \Omega$, values for $R_{P}$ range from $25 \mathrm{k} \Omega$ to $331 \mathrm{k} \Omega$. Ideality factor ranges from 5 to 17.

[1] Yang Shen, Kejia Li, Nabanita Majumdar, Joe C. Campbell, Mool C. Gupta, "Bulk and contact resistance in P3HT:PCBM heterojunction solar cells", Solar Energy Materials and Solar Cells 95, 1-4 (2011).

Keywords: Organic solar cells, electric circuit model, Lambert function.

\section{INTRODUCTION}

OSC have become one of the most interesting applications of organic electronics, as they have turn into a real alternative to inorganic solar cells with many advantages such as ease and cheapness device processing, possibility of device flexibility, and large area scalability. In the last years, a huge amount of research works have been developed with the goals of i) increasing device efficiency, ii) deepen in the understanding of physical processes responsible of electro optical conversion and iii) development of new electro optical materials. Among the new materials developed for OSC active layers, those based on blends of conjugated polymers (donor) and soluble fullerenes (acceptor) have become very popular since the discovery of efficient photoinduced charge separation at donor-acceptor interfaces. Among all materials available, blends of P3HT and PCBM are one of the most used due to the high efficiencies achieved ${ }^{1}$.

In order to design high efficiency solar cells, a good estimation of the electro optical parameters is needed. Figure 1 shows the most used equivalent circuit to model I-V curves of organic and inorganic solar cells $\mathrm{s}^{2-7}$. Mazhari et al employ a more complex model with three diodes ${ }^{8}$, which complicates not only the mathematical solution of current-voltage 
curve, but also the circuital parameters extraction. Araujo et al introduce a second diode to simulate the kink sometimes observed in the I-V characteristics of OSC due to the bad interface between cathode and polymer'

Applying Kirchhoff laws to circuit of figure 1 yields to a transcendental equation that can be solved numerically, without approximations, with the help of Lambert $\mathrm{W}$ function ${ }^{3}$. Then, using a numerical fitting procedure (least squared), parameters such as series resistance $\left(R_{S}\right)$, parallel resistance $\left(R_{P}\right)$, photogenerated current $\left(I_{p h}\right)$, ideality factor $(\eta)$ and inverse saturation current $\left(\mathrm{I}_{0}\right)$ can be extracted (this method will be called exact method, as it does not require any approximations). Other authors have simplified this task, doing some mathematical approximations and developing a simple method to obtain these parameters (this method will be called approximated method and is thoroughly explain in section 3.1). The main problem of the exact method fitting procedure encountered in this work is that the final parameters usually depend on the initial values chosen (seeds). This problem has been solved using as seeds the values obtained with the approximated method.

This work has two objectives, first the validation of the circuital model applied to OSC based on P3HT:PCBM and second to determine which parameters can be reliable using the approximation method. In order to validate the circuital model, different devices have been fabricated and their I-V curves under dark and illumination conditions have been fitted with the exact method. These fits have been performed in the forward bias region (first and fourth quadrant). From the comparison of parameters extracted from dark and illuminated curves we conclude that the model is coherent, as parameters are very similar, except from parallel resistance. This inconsistence in $R_{P}$ is due to that the range chosen for the fit is not appropriate for extracting this parameter, as its influence is only noticeable in the fourth quadrant, at very low voltage bias levels.

When comparing parameters obtained from illuminated curves using both methods (exact and approximated) results show that approximated method is good for estimating $R_{S}$ and $\eta$. The value obtained for $R_{P}$ differs in several orders of magnitude when the fit is performed in the whole range. However if the fit is done just in the fourth quadrant (where the influence of $R_{P}$ is stronger) the values obtained for $R_{P}$ are similar to those obtained with the approximated method.

This comparison has been performed using a bunch of organic solar cells based on P3HT:PCBM using three different donor/acceptor ratios and using two different speeds to deposit the active layer. This leads to different active layer thicknesses (from $100 \mathrm{~nm}$ to $250 \mathrm{~nm}$ ).

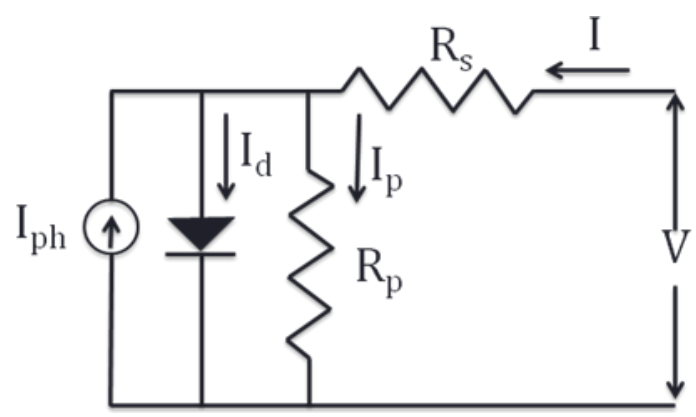

Figure 1. Conventional equivalent circuit of organic solar cells.

\section{EXPERIMENTAL TECHNIQUES}

The entire fabrication process is carried out in a clean room (class 10000). Commercial indium-tin-oxide (ITO, thickness $=100 \pm 5 \mathrm{~nm}$ ) coated glass is used as substrate. Prior to film deposition, substrates went through a typical organic material cleaning process. PEDOT:PSS was used as hole transport layer. The fabrication process consists on several steps. First, ITO coated glass is patterned by means of a photolithographic process. All layers were deposited in a glove box under $\mathrm{N}_{2}$. PEDOT:PSS is spin-coated at $5000 \mathrm{rpm}$ and dried at $110{ }^{\circ} \mathrm{C}$ for 30 minutes. A thin layer of the photoactive organic light absorber P3HT:PCBM was spin-coated at 1.000 or at $2.000 \mathrm{rpm}$ on top of the PEDOT:PSS ITO substrates. The films were fabricated with 1:1, 1:1.55 and 1:0.64 concentration ratio of P3HT:PCBM at $4 \%$ wt in anhydrous chlorobenzene. Solution underwent an ultrasound bath for several hours to get a homogeneous blend. Active 
layer was dried in an oven at $75^{\circ} \mathrm{C}$ during 2 hours. Finally the $\mathrm{Al}$ cathode is thermally evaporated on top of the organic layer surface in a $10^{-5}$ mbar atmosphere. Active area (A) is $0.07 \mathrm{~cm}^{2}$. Finally, devices were encapsulated under $\mathrm{N}_{2}$ using a glass cover attached by a bead of epoxy adhesive. A detailed description of the fabrication procedure is found in previous works ${ }^{10}$. Table 1 summarized the main characteristics of devices fabricated in this work.

Table 1. Fabrication parameters of solar cells: ratio P3HT:PCBM, spinner velocity used for active layer deposition and active layer thickness

\begin{tabular}{ccc}
\hline \hline Ratio P3HT:PCBM & Spinner velocity (r.p.m.) & Thickness (nm) \\
\hline $1: 1$ & 1000 & 275 \\
$1: 1.55$ & 1000 & 220 \\
$1: 0.64$ & 1000 & 250 \\
$1: 1$ & 2000 & 180 \\
$1: 1.55$ & 2000 & 130 \\
$1: 0.64$ & 2000 & 150 \\
\hline \hline
\end{tabular}

Thickness measurements of the polymeric active layer were performed using a DekTak 150 Veeco profilometer.

Current-voltage (I-V) characteristics were recorded using an Agilent 4155C semiconductor parameter analyzer and an Agilent 41501B SMU pulse generator. Measurements were performed (under pulsed conditions) at room temperature $\left(25^{\circ} \mathrm{C}\right)$ using a $400 \mathrm{~W} / \mathrm{m}^{2}$ halogen lamp.

\section{THEORETICAL MODEL}

Applying Kirchhoff laws to circuit of figure 1 the following equation relating current and voltage is obtained:

$$
I=I_{0}\left[e^{\frac{q}{\eta \pi T}\left(V-I R_{S}\right)}-1\right]-I_{p h}+\frac{V-I R_{S}}{R_{P}}
$$

Where $\mathrm{k}$ is Boltzmann constant, $\mathrm{q}$ is the electron charge and $\mathrm{T}$ is the temperature.

As both current and voltage appear in linear and exponential terms, an analytical expression of I (V) cannot be obtained straightforward.

\subsection{Parameters extraction with the approximation method}

In order to extract circuital parameters, many authors use standard approximations ${ }^{4,5,6,11}$. In dark conditions $\left(I_{p h}=0\right)$ and at high polarization levels, the dynamic diode resistance is low, and we can make the assumption $I_{R P}<<I_{d}$ where $I_{R P}$ and $I_{d}$ are the currents through the parallel resistance and the diode respectively. Doing this approximation, equation (1) is reduced to:

$$
I=I_{0}\left[e^{\frac{q}{\eta \pi T}\left(V-I R_{S}\right)}-1\right] \stackrel{\text { yields }}{\longrightarrow} V=\frac{\eta k T}{q} \ln \left[\frac{I}{I_{0}}+1\right]+I R_{S}
$$

Differentiating with respect to the current and assuming $\mathrm{I} \gg \mathrm{I}_{0}$ (which is valid at high polarization levels):

$$
\frac{d V}{d I}=\frac{\eta k T}{q}\left(\frac{1}{I_{/} I_{0}+1}\right) \frac{1}{I_{0}}+R_{S} \approx \frac{\eta k T}{q I}+R_{S} \Rightarrow I \frac{d V}{d I} \approx \frac{\eta k T}{q}+R_{S} I
$$

So $\mathrm{R}_{\mathrm{S}}$ can be obtained from the slope of the linear fit of $I \frac{d I}{d V}$ vs. $I$ at high current levels, and $\eta$ can be extracted from the ordinate at the origin of the linear fit. Fig. 2 shows an example of $I \frac{d I}{d V}$ vs. $I$ for diode $1: 1 / 1000 \mathrm{rpm}$. 


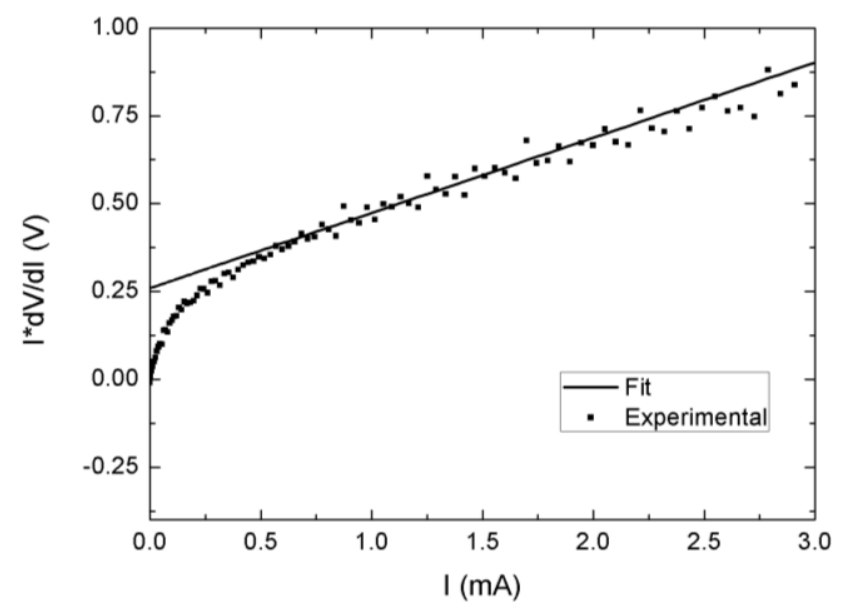

Figure 2. Experimental (dots) $I \frac{d I}{d V} v s . I$ and linear fit at high current levels (solid line).

On the other hand, assuming the dynamic diode resistance much higher than parallel resistance at very low current levels, and assuming that $\mathrm{R}_{\mathrm{P}} \gg \mathrm{R}_{\mathrm{S}}, \mathrm{R}_{\mathrm{P}}$ can be approximated by the equation:

$$
\left(\frac{d V}{d I}\right)_{V \rightarrow 0} \approx R_{S}+R_{p} \approx R_{p}
$$

After obtaining these approximated values for $\mathrm{R}_{\mathrm{P}}, \mathrm{R}_{\mathrm{S}}$ and $\eta$, and assuming $\mathrm{I}_{\mathrm{ph}} \approx \mathrm{I}_{\mathrm{SC}}, \mathrm{I}_{0}$ can be extracted from equation (1) evaluated at $\mathrm{V}=0$.

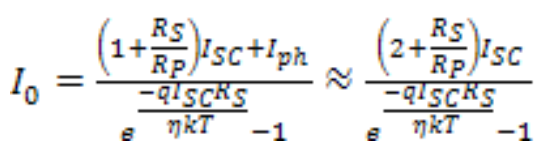

Being $\mathrm{I}_{\mathrm{SC}}$ the current evaluated at $\mathrm{V}=0$ (short circuit current).

\subsection{Parameters extraction with the exact method}

Current can be expressed as function of voltage using Lambert $\mathrm{W}$-function in equation $6^{3}$ :

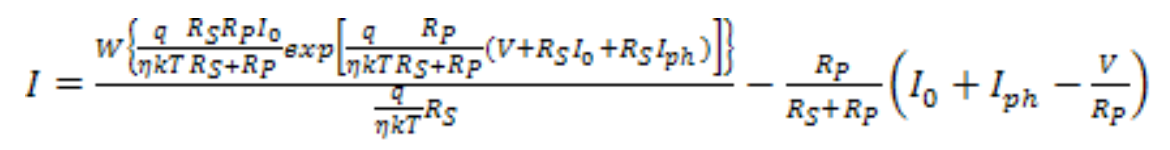

The argument of the $\mathrm{W}$-function in equation (6) only contains corresponding variable and the models parameters.

Equation (6) is fitted to the experimental I-V data using standard mathematical non linear fitting routines. We have used the non-linear curve fitting routine lsqcurvefit implemented in MATLAB, in order to find the best fitting parameters $\left(\mathrm{I}_{0}\right.$, $R_{S}, R_{P}, \eta$ and $I_{p h}$ ). This fitting process is relatively simple and just takes a few minutes to do a single current-voltage fit. Since results are seed dependent, we employ as seeds the parameters obtained with the approximated method. Fits have been performed in the forward bias regime (voltage range from $0 \mathrm{~V}$ to $2.5 \mathrm{~V}$ ). It is worth noticing that parameter values obtained may strongly depend on working voltage range.

\section{RESULTS}

\subsection{Validation of the circuital model}

In order to validate the circuit model used in this work, equation (6) has been fitted to I-V of different devices and parameters extracted from I-V curves have been compared in dark and illuminated conditions. Table 2 shows the values 
extracted: $R_{s}, R_{p}, \eta$ and $J_{0}$ (where $J_{0}=I_{0} / A$ ). The last column shows the ratio between corresponding parameter obtained from illuminated and dark curve.

As can be seen, the values of $\mathrm{R}_{\mathrm{S}}$, and $\eta$ obtained from both curves are quite similar. Inverse saturation current, $\mathrm{J}_{0}$ is of the same order of magnitude but no very similar. This difference is thought to be due to the voltage dependence of photocurrent, which is not considered in the $\operatorname{model}^{8}$. In the case of $R_{P}$, the values are very different, which is probably due to the fitting method used, least squared, and the voltage range chosen in the exact method (from $0 \mathrm{~V}$ to $2.5 \mathrm{~V}$ ).

Fig. 3 shows the fit together with the experimental data of devices with different P3HT:PCBM ratios and different active layer thicknesses at a constant illumination power of $400 \mathrm{~mW} / \mathrm{cm}^{2}$. As can be observed fits are very good especially at high currents regime.

TABLE 2. Circuital parameters extracted from illuminated and dark I-V curves fitted with the exact method. Last column shows the ratio between the corresponding parameter under illuminated and dark conditions. Fits have been performed in the whole voltage range (from 0 to $2.5 \mathrm{~V}$ ).

\begin{tabular}{|c|c|c|c||}
\hline \hline Ratio P3HT:PCBM/speed(rpm) & $\mathrm{R}_{\mathrm{S}}($ Light $)(\Omega)$ & $\mathrm{R}_{\mathrm{S}}($ Dark $)(\Omega)$ & $\frac{R_{5}(\text { Light })}{R_{5}(\text { Dark })}$ \\
\hline $1: 1 / 1000$ & 273 & 285 & 0.96 \\
\hline $1: 1.55 / 1000$ & 225 & 269 & 0.84 \\
\hline $1: 0.64 / 1000$ & 256 & 281 & 0.91 \\
\hline $1: 1 / 2000$ & 221 & 266 & 0.83 \\
\hline $1: 1.55 / 2000$ & 142 & 141 & 1.01 \\
\hline $1: 0.64 / 2000$ & 241 & 257 & 0.94 \\
\hline
\end{tabular}

\begin{tabular}{|c|c|c|c||}
\hline & & & $\frac{R_{p}(\text { Light })}{R_{p}(\text { Dark })}$ \\
\hline Ratio P3HT:PCBM/speed(rpm) & $\mathrm{R}_{\mathrm{P}}($ Light $)(\mathrm{M} \Omega)$ & $\mathrm{R}_{\mathrm{P}}($ Dark $)(\mathrm{M} \Omega)$ & 2.63 \\
\hline $1: 1 / 1000$ & 1.9 & 0.7 & 1443.30 \\
\hline $1: 1.55 / 1000$ & $1.0 \times 10^{3}$ & 0.7 & 2851.33 \\
\hline $1: 0.64 / 1000$ & $1.2 \times 10^{3}$ & 0.4 & 1.59 \\
\hline $1: 1 / 2000$ & 0.8 & 0.5 & 1845.48 \\
\hline $1: 1.55 / 2000$ & $1.3 \times 10^{3}$ & 0.7 & 0.03 \\
\hline
\end{tabular}

\begin{tabular}{|c|c|c|c||}
\hline \hline Ratio P3HT:PCBM/speed(rpm) & $\eta$ (Light) & $\eta$ (Dark) & $\frac{\eta(\text { Light) }}{\eta(\text { Dark })}$ \\
\hline $1: 1 / 1000$ & 15.3 & 14.2 & 1.08 \\
\hline $1: 1.55 / 1000$ & 10.8 & 13.8 & 0.78 \\
\hline $1: 0.64 / 1000$ & 8.9 & 9.2 & 0.97 \\
\hline $1: 1 / 2000$ & 16.7 & 13.0 & 1.29 \\
\hline $1: 1.55 / 2000$ & 5.4 & 5.5 & 0.99 \\
\hline $1: 0.64 / 2000$ & 15.6 & 13.5 & 1.16 \\
\hline
\end{tabular}




\begin{tabular}{|c|c|c|c|}
\hline \hline Ratio P3HT:PCBM/speed(rpm) & $\mathrm{J}_{0}($ Light $)\left(\mathrm{mA} / \mathrm{cm}^{2}\right)$ & $\mathrm{J}_{0}($ Dark $)\left(\mathrm{mA} / \mathrm{cm}^{2}\right)$ & $\frac{l_{0}(\text { Light })}{l_{0}(\text { Dark })}$ \\
\hline $1: 1 / 1000$ & 0.31 & 0.17 & 1.77 \\
\hline $1: 1.55 / 1000$ & 0.11 & 0.18 & 0.62 \\
\hline $1: 0.64 / 1000$ & 0.38 & 0.18 & 2.15 \\
\hline $1: 1 / 2000$ & 0.62 & 0.22 & 2.80 \\
\hline $1: 1.55 / 2000$ & 0.02 & 0.02 & 1.06 \\
\hline $1: 0.64 / 2000$ & 0.19 & 0.23 & 0.84 \\
\hline
\end{tabular}
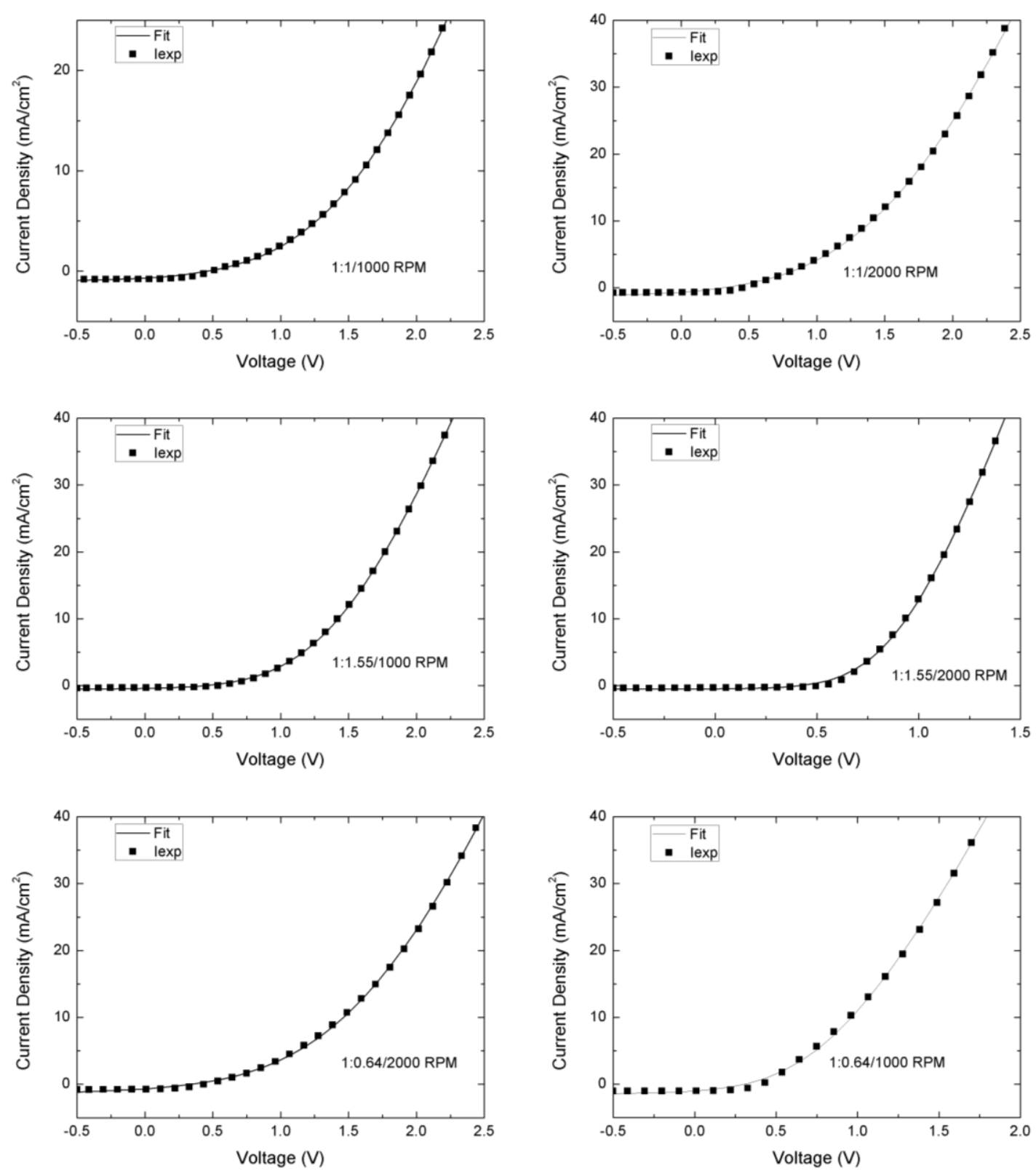

Figure 3. Experimental (filled symbols) and theoretical (solid line) J-V curves in illuminated conditions for devices with different P3HT:PCBM ratios and different deposition velocities of active layer. 
In order to validate the approximated method, parameters of every I-V curve have been extracted with the approximated method and compared to those obtained with the exact method used in this work. Table 3 summarizes these results. As can be concluded, $R_{S}$ and $\eta$, obtained with both methods are of the same order of magnitude. Maximum error between data obtained with both methods is $30 \%$. When accurate values for these parameters are needed, approximated method is not valid. On the other hand, the agreement in $R_{P}$ and $J_{0}$ is poor. It is worth mentioning that the value obtained for $R_{P}$ with the exact method may be not good since the chosen range to do the fits is not appropriate to extract $R_{P}$.

TABLE 3. Circuital parameters extracted from illuminated I-V curves fitted with the exact method and with the approximated method. Last column shows the ratio between corresponding parameter obtained with exact and approximated method. Fittings have been performed in the whole range ( 0 to $2.5 \mathrm{~V}$ )

\begin{tabular}{|c|c|c|c|}
\hline Ratio P3HT:PCBM/speed $(\mathrm{rpm})$ & $\mathrm{R}_{\mathrm{S}}($ Exact $)(\Omega)$ & $\mathrm{R}_{\mathrm{S}}($ Approximated $)(\Omega)$ & $\frac{R_{S}(\text { Exact })}{R_{S}(\text { Approximated })}$ \\
\hline $1: 1 / 1000$ & 273 & 211 & 1.29 \\
\hline $1: 1.55 / 1000$ & 225 & 208 & 1.08 \\
\hline $1: 0.64 / 1000$ & 256 & 200 & 1.28 \\
\hline $1: 1 / 2000$ & 221 & 169 & 1.31 \\
\hline $1: 1.55 / 2000$ & 142 & 118 & 1.2 \\
\hline $1: 0.64 / 2000$ & 241 & 191 & 1.26 \\
\hline
\end{tabular}

\begin{tabular}{|c|c|c|c||}
\hline \hline Ratio P3HT:PCBM/speed(rpm) & $\mathrm{R}_{\mathrm{P}}($ Exact $)(\mathrm{M} \Omega)$ & $\mathrm{R}_{\mathrm{P}}$ (Approximated) $(\mathrm{k} \Omega)$ & $\frac{R_{p} \text { (Exact) }}{R_{p}(\text { Approximated) }}$ \\
\hline $1: 1 / 1000$ & 1.89 & 40 & 47.34 \\
\hline $1: 1.55 / 1000$ & $1.0 \times 10^{3}$ & 89 & 11544.89 \\
\hline $1: 0.64 / 1000$ & $1.2 \times 10^{3}$ & 54 & 22743.94 \\
\hline $1: 1 / 2000$ & 0.77 & 91 & 8.48 \\
\hline $1: 1.55 / 2000$ & $1.3 \times 10^{3}$ & 120 & 11050 \\
\hline $1: 0.64 / 2000$ & 0.016 & 43 & 0.36 \\
\hline
\end{tabular}

\begin{tabular}{|c|c|c|c||}
\hline Ratio P3HT:PCBM/speed(rpm) & $\eta$ (Exact) & $\eta$ (Approximated) & $\frac{\eta(\text { Exact) }}{\eta(\text { Approximated) }}$ \\
\hline $1: 1 / 1000$ & 15.3 & 17.1 & 0.89 \\
\hline $1: 1.55 / 1000$ & 10.8 & 11.6 & 0.93 \\
\hline $1: 0.64 / 1000$ & 8.9 & 11.6 & 0.76 \\
\hline $1: 1 / 2000$ & 16.7 & 18.9 & 0.89 \\
\hline $1: 1.55 / 2000$ & 5.4 & 6.8 & 0.79 \\
\hline $1: 0.64 / 2000$ & 15.6 & 17.4 & 0.9 \\
\hline
\end{tabular}




\begin{tabular}{||c|c|c|c||}
\hline \hline Ratio P3HT:PCBM/speed(rpm) & $\mathrm{J}_{0}($ Exact $)\left(\mathrm{mA} / \mathrm{cm}^{2}\right)$ & $\mathrm{J}_{0}($ Approximated $)\left(\mathrm{mA} / \mathrm{cm}^{2}\right)$ & $\frac{J_{0}(\text { Exact })}{\text { lo }_{\text {Approximated })}}$ \\
\hline $1: 1 / 1000$ & 0.31 & 59.4 & $1.0 \times 10^{-2}$ \\
\hline $1: 1.55 / 1000$ & 0.11 & 41.1 & $5 . \times 10^{-3}$ \\
\hline $1: 0.64 / 1000$ & 0.38 & 41.3 & $1.8 \times 10^{-2}$ \\
\hline $1: 1 / 2000$ & 0.62 & 81.9 & $1.5 \times 10^{-2}$ \\
\hline $1: 1.55 / 2000$ & 0.02 & 42.1 & $1.2 \times 10^{-3}$ \\
\hline $1: 0.64 / 2000$ & 0.19 & 66.6 & $5.6 \times 10^{-3}$ \\
\hline
\end{tabular}

In order to calculate the $R_{P}$ accurately, another fit using the exact method has been performed in the fourth quadrant, where the role of $R_{P}$ is more significant. Results of $R_{P}$ are summarized in table 4 . As can be observed, when we reduce the voltage range, $R_{P}$ tends to the value obtained with the approximated method, as was expected. From this result we can conclude that for correctly extracting $\mathrm{R}_{\mathrm{P}}$ an adequate voltage range must be selected.

Table 4. Comparison between parallel resistances extracted by fitting the exact expression in the fourth quadrant and calculated with the approximated method. Last column shows the ratio between values obtained with both methods.

\begin{tabular}{|c|c|c|c||}
\hline \hline Ratio P3HT:PCBM/speed (rpm) & $\mathrm{R}_{\mathrm{P}}$ (Exact) $(\mathrm{k} \Omega)$ & $\mathrm{R}_{\mathrm{P}}$ (Approximated) $(\mathrm{k} \Omega)$ & $\frac{R_{p} \text { (Exact) }}{R_{P} \text { (Approximated) }}$ \\
\hline $1: 1 / 1000$ & 39 & 40 & 0.97 \\
\hline $1: 1.55 / 1000$ & 25 & 89 & 0.29 \\
\hline $1: 0.64 / 1000$ & 26 & 54 & 0.48 \\
\hline $1: 1 / 2000$ & 159 & 91 & 1.74 \\
\hline $1: 1.55 / 2000$ & 38 & 120 & 0.32 \\
\hline $1: 0.64 / 2000$ & 331 & 43 & 7.7 \\
\hline
\end{tabular}

\section{CONCLUSIONS}

In this work circuital parameters of organic solar cells based on P3HT:PCBM have been extracted with an exact method, using the analytical expression for I-V curves based on Lambert $\mathrm{W}$ function. Except from parallel resistance, all the parameters extracted from dark and illuminated curves are similar, especially $\mathbf{R}_{\mathbf{S}}$ and $\eta$. Differences found in $\mathbf{J}_{0}$ are attributed to the simplicity of the model that does not take into account the voltage dependence of photocurrent. The values obtained for $\mathrm{R}_{\mathrm{P}}$ are not reliable because of the wide range chosen to do the fits (from 0 to $2.5 \mathrm{~V}$ ).

Additionally, circuital parameters have been estimated with an approximated method for illuminated curves and have been compared with values obtained with the exact method. There is good agreement in $R_{S}$ and $\eta$ but not in $R_{P}$. However if the voltage range of the fits is restricted to the fourth quadrant, the values obtained for $R_{P}$ tend to those obtained with the approximated method.

We can conclude from these comparisons that the traditional circuit model is suitable for organic solar cells and that approximated method is good for estimating circuital parameters. However, when an accurate value is needed, exact method must be employed. Regarding parallel resistance, a narrow voltage range around $0 \mathrm{~V}$ must be chosen to properly extract this parameter, as it is in that region where the effect of $R_{\mathrm{P}}$ is significant.

\section{REFERENCES}

[1] J. Y. Kim, K. Lee, N. E. Coates, D. Moses, T.-Q. Nguyen, M. Dante, A. J. Heeger, "Efficient Tandem Polymer Solar Cells Fabricated by All-Solution Processing", Science 317, 222-225 (2007).

[2] A. Ortiz-Conde, F. J. García Sánchez, J. Mucci, "New method to extract the model parameters of solar cells from the explicit analytic Solutions of their illuminated I-V characteristics", Solar Energy Materials and Solar Cells 90, 352-361 (2006). 
[3] A. Jain, A. Kapoor, “A new approach to study organic solar cell using Lambert W-function”, Solar Energy Materials and Solar Cells 86, 197-205 (2005).

[4] S. Yoo, B. Domercq, and B. Kippelen, "Intensity-dependent equivalent circuit parameters of organic solar cells based on pentacene and C60", J. Appl. Phys. 97, 103706 (2005).

[5] K. Ishibashi, Y. Kimura and M. Niwano, "An extensively valid and stable method for derivation of all parameters of a solar cell from a single current-voltage characteristic", J. Appl. Phys. 103, 094507 (2008).

[6] C. Zhang, J. Zhang, Y. Hao, Z. Lin, and C. Zhu, "A simple and efficient solar cell parameter extraction method from a single current-voltage curve”, J. Appl. Phys. 110, 064504 (2011).

[7] S. Lattante, A. Perulli and M Anni, "Study of the series resistance evolution in organic solar cells by use of the Lambert W function", Synth Met. 161, 949-952 (2011).

[8] B. Mazhari, “An improved solar cell circuit model for organic solar cells”, Solar Energy Materials and Solar Cells 90, 1021-1033 (2006).

[9] F. Araujo de Castro, J. Heier, F. Nüesch and R. Hany, "Origin of the kink in current-density versus voltage curves and efficiency enhancement of polymer-C60 heterojunction solar cells", IEEE Journal of Select Topics in Quantum Electronics 16, 1690-1699 (2010).

[10] Romero B, Arredondo B, Alvarez AL, Mallavia R, Salinas A, Quintana BX, et al. "Influence of Electrical Operating Conditions and Active Layer Thickness on Electroluminescence Degradation in Polyfluorene-phenylene based Light Emitting Diodes", Solid-State Electron 53, 211 (2009).

[11] Ali Cheknane, Hikmat S. Hilal, Fayc- al Djeffal, Boumediene Benyoucef, Jean-Pierre Charles, “An equivalent circuit approach to organic solar cell modelling”, Microelectronics Journal 39, 1173-1180 (2008). 